ABSTRACT ID: 8

Poster(Competing)

\title{
Evaluation Of Knowledge And Attitude Towards Oral Health During Pregnancy And Common Practices Among Pregnant Women Residing In Pahang, Malaysia
}

Nurjasmine Aida Jamani ${ }^{\mathrm{a}}$ | Karimah Hanim Abdul Aziz ${ }^{\mathrm{b}}$ | Zurainie Abllah ${ }^{\mathrm{c}}$

${ }^{a}$ Department of Family Medicine, International Islamic University Malaysia $\left.\right|^{b}$ Department of Community Medicine, International Islamic University Malaysia I 'Department of Paediatric Dentistry, Orthodontics and Dental Public Health, Kulliyyah of Dentistry, International Islamic University Malaysia

Introduction: Oral health problem among pregnant woman has been shown to have possible association with preterm birth and low birth weight. Several studies found that pregnant women have limited knowledge of these adverse outcome. The aims of this study were to evaluate the knowledge, attitude and practices of pregnant women and to examine their practices in relation with their intraoral health status. Materials and Methods: This a cross-sectional study where pregnant women from government health clinics in Kuantan, Pahang completed a questionnaire to assess their knowledge, attitudes and practices towards oral health. A thorough dental examination was done to attain their oral health status. Results were analyzed by descriptive analysis, chi square test and multiple logistic regressions. Results: 296 pregnant women participated in this study. The mean age of the respondents was 28.7 years and the mean parity was two (2). Majority of them were Malay (91.9\%) and had education below than higher secondary school (47.3\%). Most respondents demonstrated poor knowledge $(67 \%)$ and poor attitude $(54 \%)$ about dental health in general.175 $(59.1 \%)$ respondents do not know that gum disease are common in pregnancy and 235 (79.4\%) of them are unaware of gum problems causing possible risk of adverse outcome in pregnancy. Half of them (53\%) had good practice on oral health. Women with increase parity and are working were less likely to develop missing teeth. Good attitude towards dental health was found to be a protective factor for oral health disease. Conclusion(s): Most women were less knowledgeable and had poor attitude about oral and dental health. Gestation, employment and good attitude were significantly associated with good oral health outcome. Hence, targeted education should be promoted during pre-pregnancy in order to prevent possible adverse pregnancy outcome.

KEYWORDS: oral health, knowledge, practice, pregnant women 